\section{PTU-041 ‘ ‘REALLY DOCTOR! I HAVE COELIAC DISEASE? THAT'S OK I'M WEALTHY AND UNIVERSITY EDUCATED'}

doi:10.1136/gut.2011.239301.169

S M Barratt, ${ }^{*}$ A Arnaout, K E Evans, J S Leeds, D S Sanders The GI \& Liver Unit, Sheffield Teaching Hospitals, Sheffield, UK

Introduction The gluten-free diet (GFD) is the mainstay of treatment in Coeliac Disease, however, this diet is potentially very difficult to follow presenting an array of obstacles. Due to such difficulties some patients do not consistently adhere to a GFD, or do not follow a GFD at all. We aimed to determine what patient characteristics are associated with sub-optimal GFD adherence. In doing so we hoped to identify poor prognostic indicators of treatment compliance.

Methods Postal survey of 225 histologically proven Coeliac Disease patients $(5 \% 18-25$ years, $4 \% 26-35$ years, $18 \% 36-45$ years, $19 \% 46-55$ years, $26 \% 56-65$ years, $28 \%>66$ years) of which $25 \%$ were male. Mean disease duration 8 years, range 0.5-51years. Questionnaire included age, sex, highest educational qualification and marital status. Postcodes were used to assign A.C.O.R.N. categories (A Classification of Residential
Neighbourhoods). These categories are based on geo-demographic variables including income, housing and government census and lifestyle survey data. Medical records were used to determine disease duration and method of presentation at diagnosis (Typical: GI symptoms; Atypical: non-GI symptoms; Screen-detected: asymptomatic). Patients stated their level of GFD adherence in the previous 28 days: Full Adherence (FA): everyday with no dietary transgressions, or Partial/None Adherence (PNA): less than FA.

Results Age was not a confounding factor. ACORN groups other than 'Wealthy Executives' had a reduced likelihood of FA (OR 0.33, 95\% CI 0.15 to 0.75, p=0.0077). Deprived ACORN groups 'Hard Pressed' and 'Moderate Means' had a reduced likelihood of FA compared to the affluent 'Comfortably Off', 'Urbanites' and 'Wealthy Executives' (OR $0.32,95 \%$ CI 0.14 to $0.70, p=0.0057) .28 \%$ of FA patients held a university degree versus $16 \%$ of PNA patients ( $p=0.064$ ). $25 \%$ of FA and $24 \%$ of PNA patients were male ( $p=n s) .72 \%$ of FA and $71 \%$ of PNA patients were married ( $p=n s$ ). $4 \%$ of FA and $12 \%$ of PNA patients were under 35 years of age ( $p=0.085) .26 \%$ of FA and $31 \%$ of PNA patients had a disease duration of three years or less ( $p=n s) .13 \%$ of FA and $21 \%$ of PNA patients had a screendetected method of presentation ( $\mathrm{p}=\mathrm{ns}$ ).

Conclusion Poor socio-economic background and educational achievement that is below university (i.e. degree) level are associated with reduced or absent GFD adherence. This suggests wealthier patients can 'buy' greater food choices to accommodate this restrictive diet and higher educational achievement may promote greater awareness of the diet and strategies to overcome problems. A disease duration less than three years and being under 35 years of age are also associated with reduced GFD adherence, but to a statistically insignificant level. Our findings allow professionals to identify 'at risk' patients in Coeliac Disease.

Competing interests None.

Keywords Coeliac Disease, Demographics, Gluten free diet, Health Inequalities. 